\title{
Systemic Circulatory Response to Stress of Simulated Flight and to Physical Exercise Before and After Propranolol Blockade*
}

\author{
HARALD ELIASCH, ANDERS ROSÉN, AND HUGH M. SCOTT \\ From the Department of Medicine, Karolinska Institutet at the Serafimer Hospital, and the Medical Division, \\ the Royal Swedish Airforce, Stockholm, Sweden
}

Stress can induce profound responses in the cardiovascular system. In such situations increased systemic blood pressure and flow have been observed (Grollman, 1929; Stead et al., 1945; Hickam, Cargill, and Golden, 1948; Stevenson, Duncan, and Wolff, 1949; Wolf et al., 1955). Much of our further knowledge of the responses to emotional stimuli on central and regional hæmodynamics in man has been summarized by Brod (1963).

In most investigations the experimental situations and environments cannot be considered representative of day-to-day stressful occurrences. For example, most often stimuli such as mental arithmetic and/or cold pressure tests have been applied at the bedside or in a laboratory setting. In addition, studies have been made on subjects lying, whereas upright circulatory adjustments should be of more practical interest.

The mechanisms underlying the cardiovascular responses to stress have also been studied (Brod et al., 1959; Blair et al., 1959). While it is generally believed that the sympatho-adrenal system plays a dominant role in mediating the cardiovascular responses to emotional stress, it has been difficult to appraise quantitatively the contribution made by the adrenergic system, and more specifically the portion mediated by the beta adrenergic receptors (Ahlquist, 1948).

The present report describes an experimental model that attempts to overcome some of the limitations mentioned, and also to analyse the contribution of sympathetic cardiac stimulation and adrenergic vasodilation to the circulatory reactions caused

Received June 15, 1966.

* Presented in part at the 38th Scientific Sessions of the American Heart Association, Bal Harbour, Miami Beach, Florida, October 1965. by stress. Heart rate, cardiac output, systemic blood pressure, and plasma catecholamines have been measured in pilots performing Link trainer simulated flight. Thus, a difficult yet familiar task to the subjects' daily occupation, carried through in the sitting posture, is introduced as a suitable stressor. In a number of subjects, the hæmodynamic responses to a standardized exercise test have been determined in order to compare these responses with those to emotional stress. Such simultaneous studies have only occasionally been performed in earlier investigations (Brod, 1963). Finally, beta adrenergic receptor blockade by the use of propranolol (Black et al., 1964; Black, Duncan, and Shanks, 1965) has been applied in both of these situations in an attempt to analyse the degree of involvement of this receptor system.

\section{SUBJECTS AND METHODS}

The results of the study are based on observations made in 15 pilots (Table I), all men, and selected on a volunteer basis. In 9 (No. 1-2, 4-10) a prize was offered for the best performance. These 9 subjects as well as No. 14 and 17, all varying in age from 22 to 49 years, were in good health as judged from annual medical examinations, and they had displayed no symptoms or signs of cardiovascular disease. Subject No. 3, a commercial airline pilot, aged 46, had been in hospital for arterial hypertension, with an admission pressure of $220 / 130 \mathrm{~mm}$. Hg. Subsequent examination in this man, including renal aortography, showed no signs of secondary vascular involvement. Two pilots (No. 13 and 15) aged 30 and 43, respectively, had on occasional examinations displayed transitory blood pressure rises. Subject No. 11, an Air Force officer, aged 46, showed atypical S-T changes on his electrocardiogram. He had no symptoms of cardiovascular disease and his physical working capacity revealed no evidence of vascular in671 


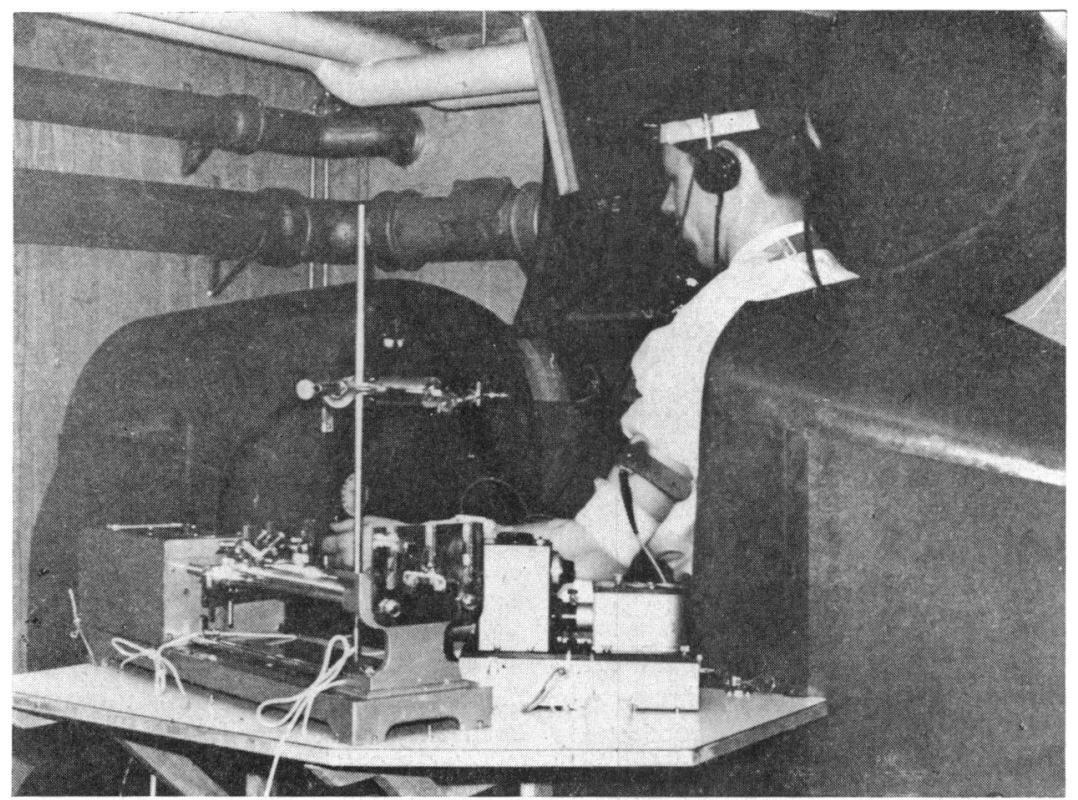

FIG. 1.-The pilot is seated in a Link trainer, with his left arm resting outside on a platform, attached to the cabin. The strain gauge manometer and part of the equipment used for dye dilution arterial withdrawal are mounted.

The hæmodynamic response to the flight procedure was studied in all pilots. The response to a second flight following the injection of saline was investigated in four pilots (No. 1-4). The response to a second flight following the injection of propranolol was studied in the remaining 11 pilots (No. 5-15). Finally, in addition to flight in five of these pilots (No. 11-15) the response to physical exercise was also studied both before and after the administration of propranolol.

Originally, 18 pilots volunteered for the investigation. As one of them had a vasovagal syncopal attack at the start of the first exercise, and technical difficulties interfered with cardiac output determinations in two others, these three pilots were excluded from this report.

Teflon catheters were introduced percutaneously in the cubital artery and an antecubital vein of the left arm in each subject. The tip of the venous catheter was advanced $30 \mathrm{~cm}$. to the superior vena cava.

After one hour at rest the subject was seated in a Link trainer, used for routine flight training, with the left arm resting comfortably outside on a platform attached to the cabin (Fig. 1). Intra-arterial blood pressure was sensed by a strain gauge manometer and together with one electrocardiographic lead recorded on an ink writing oscillograph (Elema, Mingograph 42). The manometer was level with the xiphoid notch in all situations. Cardiac output was measured by dye dilution arterial withdrawal through a Gilford Cuvette densitometer 103 IR after the injection of $5 \mathrm{mg}$. indocyanine green into the venous catheter, the resultant dye curve being inscribed on a Kipp Micrograph BD 2.
Arterial samples $(16 \mathrm{ml}$.) for catecholamine analyses were taken before and during flight in 10 pilots, and before and during exercise in 5 pilots (Table I). The modified trihydroxyindole method for the determination of epinephrine and norepinephrine in plasma described by Häggendal (1963) was used. Dimercaptopropanol (BAL) in sodium sulphite solution was thus substituted for ascorbic acid. The recovery of added epinephrine and norepinephrine $(5-25 \mu \mathrm{g}$. to $10 \mathrm{ml}$. plasma) was $73 \pm 9$ and $80 \pm 6$ per cent (mean \pm S.E.), respectively. The method does not allow for measurement of amine concentrations equal to or below $0.1 \mu \mathrm{g}$. per litre plasma. The figures given in the text are not corrected for the loss in the methodological procedure.

Part of the equipment used for the measurement of pressure and flow was placed on the platform described above, which moved with the Link trainer during the flight. Appropriate measures were taken to counterbalance the added weight. The top of the cockpit was closed so that the subject was unaware of the activities of the investigators (Fig. 2). The pilot communicated with a flight instructor via a radiotelephone connexion.

The exercise test was performed in the sitting posture on a bicycle ergometer (Holmgren, 1956).

Propranolol*, a specific beta adrenergic receptor blocking agent with no apparent sympathomimetic properties (Black et al., 1965), was infused intravenously in 11 subjects.

* The drug was kindly supplied by its manufacturer, Imperial Chemical Industries Limited. 


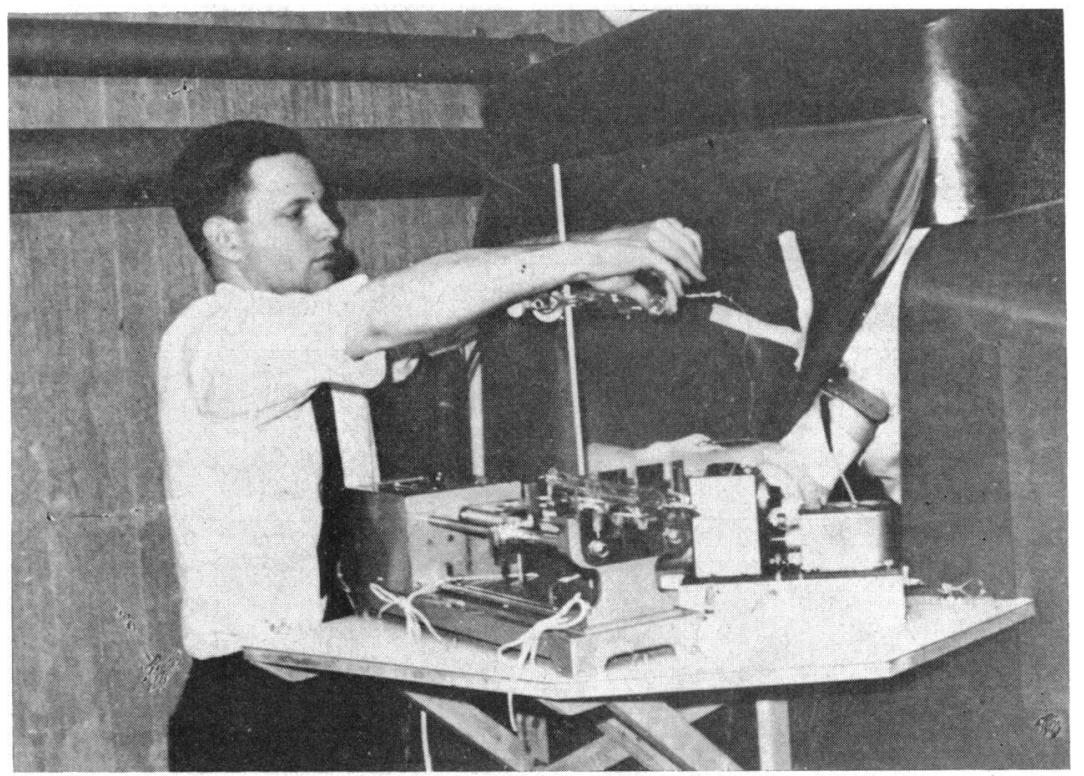

FIG. 2.-The cabin door is replaced by a thick apron allowing for sufficient closure of the cockpit.

In the initial portion of each study all subjects were investigated in the same manner. First, the heart rate and blood pressure response to two intravenous injections of isoprenaline (4-6 $\mu \mathrm{g}$.), given 5 minutes apart, were recorded. During a subsequent period lasting 15 minutes, while the pilot was sitting quietly in the trainer, intermittent recordings of arterial blood pressure and heart rate were made. Duplicate cardiac output measurements were made, and an arterial sample for the determination of plasma catecholamines was taken.

An instructional period followed the resting period. The Link trainer was released and the pilot went through simple familiarization manœuvres. Heart rate, arterial blood pressure, and duplicate cardiac output measurements were again recorded. After this, the pilot was directed by the instructor throughout a navigation flight period which was introduced and concluded acutely. His ability to retain course, speed, altitude, and time was evaluated. During the flight, intermittent mental arithmetic problems were introduced. Questions pertaining to navigation were asked to simulate ordinarily occurring distractions during flight and the number of erroneous answers recorded. Measurements were made in a similar fashion as during previous periods and a further sample for catecholamine analysis was taken. The pilots were then observed throughout a recovery period of 15 minutes during which arterial pressure and heart rate were recorded and duplicate cardiac outputs measured.

The procedure was varied at this point as follows.

(a) In four subjects (No. 1-4) $5 \mathrm{ml}$. saline was infused intravenously throughout five minutes. Further resting readings were taken, a second flight was introduced acutely, and identical studies were made to those of the first flight period.

(b) In six pilots (No. 5-10) $5 \mathrm{mg}$. propranolol was infused intravenously throughout 5 minutes. The infusion was followed by a second rest and flight period, respectively, as described under the previous section. The second recovery period was concluded with two further injections of isoprenaline, in the same dose that was given initially, to study the heart and blood pressure responses after the administration of propranolol.

(c) Five pilots (No. 11-15) left the Link trainer and were seated on a bicycle ergometer, immediately starting exercise. The load was $600 \mathrm{kpm} / \mathrm{min}$. for a period of 6 minutes. Heart rate, blood pressure, and cardiac output were measured and arterial samples for catecholamine determination were taken before and during the exercise period. Immediately thereafter, the pilot was seated again in the Link trainer and $5 \mathrm{mg}$. propranolol was infused during five minutes and the whole experimental procedure with the flight and exercise was repeated. This time, a second effort period with a load of 900 $\mathrm{kpm} / \mathrm{min}$. was added to the exercise performance. When back in the Link trainer after the exercise, two further injections of isoprenaline in the doses given initially were administered, while heart rate and blood pressure were recorded.

The arterial blood withdrawn for the dye dilution technique was reinjected at all times. Blood loss, resulting mainly from arterial sampling for analysis of catecholamines, did not exceed $120 \mathrm{ml}$. in each study.

The heart rate increased on an average by 41 per cent following the two consecutive i.v. injections of $4-6 \mu \mathrm{g}$. isoprenaline, administered at the beginning of the study. On the two similar injections, given after the propranolol flight or exercise test, the rise in heart rate did not exceed 6 beats a minute. The diastolic blood pressure measured on identical occasions as described above decreased on an average by 36 per cent before and by 3 per cent after the administration of propranolol. 
TABLE I

HAMODYNAMIC STUDIES BEFORE AND AFTER I.V. ADMINISTRATION OF $5 \mathrm{ML}$. ISOTONIC SALINE (NO. 1-4), AND 5 MG. PROPRANOLOL (NO. 5-15)

\begin{tabular}{|c|c|c|c|c|c|c|c|c|c|c|c|c|c|}
\hline \multirow[t]{2}{*}{$\begin{array}{l}\text { Subject } \\
\text { No. }\end{array}$} & \multirow[t]{2}{*}{$\begin{array}{l}\text { Age } \\
\text { (yr.) }\end{array}$} & \multirow[t]{2}{*}{$\underset{\left(\mathrm{m}^{2}\right)}{\mathrm{BSA}}$} & \multirow[t]{2}{*}{ Procedure } & \multirow[t]{2}{*}{$\begin{array}{c}\text { Heart } \\
\text { ratel } \\
\text { min. }\end{array}$} & \multicolumn{3}{|c|}{$\begin{array}{c}\text { Brachial arterial } \\
\text { pressure (mm. Hg) }\end{array}$} & \multirow[t]{2}{*}{$\begin{array}{c}\text { Cardiac } \\
\text { output } \\
\text { (1./min.) }\end{array}$} & \multirow[t]{2}{*}{$\begin{array}{c}\text { Stroke } \\
\text { volume } \\
(\mathrm{ml} .)\end{array}$} & \multirow{2}{*}{$\begin{array}{l}\text { Total } \\
\text { periph. } \\
\text { vasc. } \\
\text { resist. }\end{array}$} & \multicolumn{2}{|c|}{ 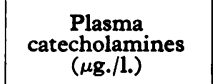 } & \multirow[t]{2}{*}{$\begin{array}{l}\text { Flight } \\
\text { errors }\end{array}$} \\
\hline & & & & & Syst. & Diast. & Mean & & & & Epineph. & $\begin{array}{c}\begin{array}{c}\text { Nor- } \\
\text { epineph. }\end{array} \\
\text {. }\end{array}$ & \\
\hline \multirow[t]{2}{*}{1} & \multirow[t]{2}{*}{21} & \multirow[t]{2}{*}{$1 \cdot 80$} & \multirow{2}{*}{$\begin{array}{l}\text { Rest } \\
\text { Flight } \\
\text { Recovery } \\
\text { Saline } \\
\text { Rest } \\
\text { Flight } \\
\text { Recovery }\end{array}$} & $\begin{array}{l}55 \\
71 \\
59\end{array}$ & $\begin{array}{l}131 \\
165 \\
164\end{array}$ & $\begin{array}{l}80 \\
94 \\
89\end{array}$ & $\begin{array}{l}102 \\
128 \\
119\end{array}$ & $\begin{array}{l}4 \cdot 6 \\
8 \cdot 1 \\
5 \cdot 4\end{array}$ & $\begin{array}{r}84 \\
110 \\
99\end{array}$ & $\begin{array}{l}2 \cdot 2 \\
1 \cdot 6 \\
2 \cdot 2\end{array}$ & $\leqq 0.1$ & $\begin{array}{l}0.4 \\
0.7\end{array}$ & \multirow[t]{2}{*}{1} \\
\hline & & & & $\begin{array}{l}54 \\
66 \\
51\end{array}$ & $\begin{array}{l}154 \\
160 \\
154\end{array}$ & $\begin{array}{l}81 \\
84 \\
79\end{array}$ & $\begin{array}{l}113 \\
112 \\
106\end{array}$ & $\begin{array}{l}5 \cdot 4 \\
6.8 \\
4.5\end{array}$ & $\begin{array}{l}92 \\
98 \\
89\end{array}$ & $\begin{array}{l}2 \cdot 2 \\
1 \cdot 7 \\
2 \cdot 3\end{array}$ & & & \\
\hline \multirow[t]{2}{*}{2} & \multirow[t]{2}{*}{26} & \multirow[t]{2}{*}{$1 \cdot 76$} & \multirow{2}{*}{$\begin{array}{l}\text { Rest } \\
\text { Flight } \\
\text { Recovery } \\
\text { Saline } \\
\text { Rest } \\
\text { Flight } \\
\text { Recovery } \\
\end{array}$} & $\begin{array}{l}76 \\
87 \\
77\end{array}$ & $\begin{array}{l}146 \\
162 \\
144\end{array}$ & $\begin{array}{l}82 \\
96 \\
84\end{array}$ & $\begin{array}{l}108 \\
119 \\
104\end{array}$ & \multirow[t]{2}{*}{$\begin{array}{l}5 \cdot 8 \\
7 \cdot 0 \\
4 \cdot 6\end{array}$} & \multirow[t]{2}{*}{$\begin{array}{l}76 \\
82 \\
60\end{array}$} & \multirow[t]{2}{*}{$\begin{array}{l}1 \cdot 9 \\
1.7 \\
2 \cdot 2\end{array}$} & & & \multirow{2}{*}{$\begin{array}{l}1 \\
3\end{array}$} \\
\hline & & & & $\begin{array}{l}74 \\
80 \\
70\end{array}$ & $\begin{array}{l}138 \\
146 \\
135\end{array}$ & $\begin{array}{l}80 \\
89 \\
78\end{array}$ & $\begin{array}{l}100 \\
111 \\
102\end{array}$ & & & & & & \\
\hline \multirow[t]{2}{*}{3} & \multirow[t]{2}{*}{46} & \multirow[t]{2}{*}{$2 \cdot 00$} & \multirow{2}{*}{$\begin{array}{l}\text { Rest } \\
\text { Flight } \\
\text { Recovery } \\
\text { Saline } \\
\text { Rest } \\
\text { Flight } \\
\text { Recovery }\end{array}$} & $\begin{array}{l}63 \\
88 \\
64\end{array}$ & $\begin{array}{l}170 \\
216 \\
198\end{array}$ & $\begin{array}{l}108 \\
128 \\
113\end{array}$ & $\begin{array}{l}137 \\
162 \\
141\end{array}$ & $\begin{array}{l}4 \cdot 0 \\
7 \cdot 7 \\
4 \cdot 4\end{array}$ & $\begin{array}{l}65 \\
81 \\
71\end{array}$ & $\begin{array}{l}3 \cdot 4 \\
2 \cdot 1 \\
3 \cdot 2\end{array}$ & & & \\
\hline & & & & $\begin{array}{l}64 \\
84 \\
62\end{array}$ & $\begin{array}{l}205 \\
225 \\
195\end{array}$ & $\begin{array}{l}116 \\
127 \\
114\end{array}$ & $\begin{array}{l}147 \\
169 \\
145\end{array}$ & $\begin{array}{l}4 \cdot 1 \\
7 \cdot 8 \\
4 \cdot 6\end{array}$ & $\begin{array}{l}67 \\
92 \\
77\end{array}$ & $\begin{array}{l}3 \cdot 6 \\
2 \cdot 2 \\
3 \cdot 4\end{array}$ & & & \\
\hline \multirow[t]{2}{*}{4} & 27 & $2 \cdot 12$ & $\begin{array}{l}\text { Rest } \\
\text { Flight } \\
\text { Recovery } \\
\text { Saline }\end{array}$ & $\begin{array}{l}66 \\
85 \\
68\end{array}$ & $\begin{array}{l}109 \\
145 \\
131\end{array}$ & $\begin{array}{l}61 \\
83 \\
74\end{array}$ & $\begin{array}{r}78 \\
110 \\
96\end{array}$ & $\begin{array}{r}7 \cdot 4 \\
12 \cdot 8 \\
9 \cdot 1\end{array}$ & $\begin{array}{l}112 \\
149 \\
129\end{array}$ & $\begin{array}{l}1.1 \\
0.9 \\
1.1\end{array}$ & $\begin{array}{l}\leqq 0 \cdot 1 \\
\leqq 0 \cdot 1\end{array}$ & $\begin{array}{l}0.4 \\
0.4\end{array}$ & 1 \\
\hline & & & $\begin{array}{l}\text { Rest } \\
\text { Flight } \\
\text { Recovery }\end{array}$ & $\begin{array}{l}68 \\
85 \\
69\end{array}$ & $\begin{array}{l}118 \\
142 \\
130\end{array}$ & $\begin{array}{l}70 \\
84 \\
74\end{array}$ & $\begin{array}{r}87 \\
105 \\
97\end{array}$ & $\begin{array}{r}8 \cdot 0 \\
13 \cdot 3 \\
9 \cdot 1\end{array}$ & $\begin{array}{l}121 \\
149 \\
144\end{array}$ & $\begin{array}{l}1 \cdot 1 \\
0 \cdot 8 \\
1 \cdot 1\end{array}$ & & & 1 \\
\hline 5 & 22 & 1.87 & $\begin{array}{l}\text { Rest } \\
\text { Flight } \\
\text { Recovery }\end{array}$ & $\begin{array}{l}69 \\
80 \\
74\end{array}$ & $\begin{array}{l}135 \\
155 \\
147\end{array}$ & $\begin{array}{l}78 \\
94 \\
89\end{array}$ & $\begin{array}{l}101 \\
116 \\
117\end{array}$ & $\begin{array}{l}3.9 \\
5.2 \\
4 \cdot 4\end{array}$ & $\begin{array}{l}59 \\
62 \\
57\end{array}$ & $\begin{array}{l}2 \cdot 7 \\
2 \cdot 3 \\
2 \cdot 7\end{array}$ & $\begin{array}{l}\leqq \\
\leqq 0 \cdot 1\end{array}$ & $\begin{array}{l}0.8 \\
0.5\end{array}$ & 2 \\
\hline & & & $\begin{array}{l}\text { Rest } \\
\text { Flight } \\
\text { Recovery }\end{array}$ & $\begin{array}{l}56 \\
65 \\
52 \\
\end{array}$ & $\begin{array}{l}140 \\
150 \\
135 \\
\end{array}$ & $\begin{array}{l}89 \\
99 \\
81\end{array}$ & $\begin{array}{l}113 \\
124 \\
110 \\
\end{array}$ & $\begin{array}{l}2.9 \\
3 \cdot 1 \\
2.5\end{array}$ & $\begin{array}{l}53 \\
46 \\
50\end{array}$ & $\begin{array}{l}3.9 \\
4 \cdot 0 \\
4 \cdot 3\end{array}$ & & & 1 \\
\hline 6 & 22 & $1 \cdot 71$ & $\begin{array}{l}\text { Rest } \\
\text { Flight } \\
\text { Recovery }\end{array}$ & $\begin{array}{l}67 \\
85 \\
69\end{array}$ & $\begin{array}{l}135 \\
151 \\
152\end{array}$ & $\begin{array}{l}79 \\
98 \\
95\end{array}$ & $\begin{array}{l}102 \\
122 \\
122\end{array}$ & $\begin{array}{l}5 \cdot 4 \\
7 \cdot 1 \\
5 \cdot 8\end{array}$ & $\begin{array}{l}83 \\
86 \\
88\end{array}$ & $\begin{array}{l}1.9 \\
1.7 \\
2 \cdot 1\end{array}$ & $\leqq 0 \cdot 1$ & $\begin{array}{l}0.3 \\
0.9\end{array}$ & 3 \\
\hline & & & $\begin{array}{l}\text { Rest } \\
\text { Flight } \\
\text { Recovery }\end{array}$ & $\begin{array}{l}56 \\
59 \\
52\end{array}$ & $\begin{array}{l}152 \\
156 \\
146\end{array}$ & $\begin{array}{r}95 \\
101 \\
87\end{array}$ & $\begin{array}{l}119 \\
124 \\
114\end{array}$ & $\begin{array}{l}4 \cdot 7 \\
4 \cdot 8 \\
4 \cdot 1\end{array}$ & $\begin{array}{l}82 \\
81 \\
84\end{array}$ & $\begin{array}{l}2 \cdot 5 \\
2 \cdot 6 \\
2 \cdot 8\end{array}$ & & & 1 \\
\hline 7 & 31 & $1 \cdot 78$ & $\begin{array}{l}\text { Rest } \\
\text { Flight } \\
\text { Recovery } \\
\text { Propranolol }\end{array}$ & $\begin{array}{r}86 \\
120 \\
96\end{array}$ & $\begin{array}{l}165 \\
187 \\
182\end{array}$ & $\begin{array}{l}103 \\
119 \\
117\end{array}$ & $\begin{array}{l}128 \\
143 \\
144\end{array}$ & $\begin{array}{r}8 \cdot 0 \\
14 \cdot 0 \\
10 \cdot 2\end{array}$ & $\begin{array}{r}97 \\
116 \\
107\end{array}$ & $\begin{array}{l}1.6 \\
1.0 \\
1.4\end{array}$ & $\begin{array}{l}1.7 \\
1.4\end{array}$ & $\begin{array}{l}1.4 \\
1.7\end{array}$ & 1 \\
\hline & & & $\begin{array}{l}\text { Rest } \\
\text { Flight } \\
\text { Recovery }\end{array}$ & $\begin{array}{l}72 \\
80 \\
63\end{array}$ & $\begin{array}{l}189 \\
194 \\
166\end{array}$ & $\begin{array}{l}125 \\
130 \\
111\end{array}$ & $\begin{array}{l}153 \\
156 \\
136\end{array}$ & $\begin{array}{l}6 \cdot 5 \\
6 \cdot 7 \\
5 \cdot 6\end{array}$ & $\begin{array}{l}89 \\
86 \\
91\end{array}$ & $\begin{array}{l}2 \cdot 4 \\
2 \cdot 3 \\
2 \cdot 4\end{array}$ & & & 1 \\
\hline 8 & 24 & 1.91 & $\begin{array}{l}\text { Rest } \\
\text { Flight } \\
\text { Recovery } \\
\text { Propranolol }\end{array}$ & $\begin{array}{r}83 \\
107 \\
83\end{array}$ & $\begin{array}{l}133 \\
155 \\
137\end{array}$ & $\begin{array}{r}89 \\
106 \\
95\end{array}$ & $\begin{array}{l}109 \\
124 \\
113\end{array}$ & $\begin{array}{l}5 \cdot 6 \\
9 \cdot 3 \\
5 \cdot 7\end{array}$ & $\begin{array}{l}71 \\
86 \\
71\end{array}$ & $\begin{array}{l}1.9 \\
1.3 \\
2.0\end{array}$ & $\leqq 0 \cdot 1$ & $\begin{array}{l}1.2 \\
1.2\end{array}$ & 3 \\
\hline & & & $\begin{array}{l}\text { Rest } \\
\text { Flight } \\
\text { Recovery }\end{array}$ & $\begin{array}{l}68 \\
75 \\
69\end{array}$ & $\begin{array}{l}136 \\
153 \\
137\end{array}$ & $\begin{array}{r}93 \\
108 \\
95\end{array}$ & $\begin{array}{l}110 \\
125 \\
111\end{array}$ & $\begin{array}{l}4 \cdot 1 \\
5 \cdot 8 \\
4 \cdot 6\end{array}$ & $\begin{array}{l}61 \\
74 \\
70\end{array}$ & $\begin{array}{l}2 \cdot 7 \\
2 \cdot 2 \\
2 \cdot 5\end{array}$ & & & 2 \\
\hline 9 & 24 & 1.97 & $\begin{array}{l}\text { Rest } \\
\text { Flight } \\
\text { Recovery }\end{array}$ & $\begin{array}{l}78 \\
91 \\
77\end{array}$ & $\begin{array}{l}122 \\
156 \\
144\end{array}$ & $\begin{array}{l}78 \\
93 \\
88\end{array}$ & $\begin{array}{r}98 \\
121 \\
112\end{array}$ & $\begin{array}{l}5 \cdot 4 \\
9 \cdot 4 \\
6 \cdot 4\end{array}$ & $\begin{array}{r}76 \\
102 \\
85\end{array}$ & $\begin{array}{l}1 \cdot 7 \\
1 \cdot 3 \\
1.7\end{array}$ & & & 3 \\
\hline & & & $\begin{array}{l}\text { Rest } \\
\text { Flight } \\
\text { Recovery }\end{array}$ & $\begin{array}{l}65 \\
68 \\
64\end{array}$ & $\begin{array}{l}131 \\
155 \\
133\end{array}$ & $\begin{array}{r}82 \\
102 \\
83\end{array}$ & $\begin{array}{l}105 \\
124 \\
109\end{array}$ & $\begin{array}{l}4 \cdot 5 \\
4 \cdot 8 \\
5 \cdot 1\end{array}$ & $\begin{array}{l}70 \\
73 \\
81\end{array}$ & $\begin{array}{l}2 \cdot 3 \\
2 \cdot 6 \\
2 \cdot 1\end{array}$ & & & 3 \\
\hline
\end{tabular}

Table I cont'd. 
TABLE I continued

\begin{tabular}{|c|c|c|c|c|c|c|c|c|c|c|c|c|c|}
\hline \multirow[t]{2}{*}{$\begin{array}{l}\text { Subject } \\
\text { No. }\end{array}$} & \multirow[t]{2}{*}{$\begin{array}{l}\text { Age } \\
\text { (yr.) }\end{array}$} & \multirow[t]{2}{*}{$\underset{\left(\mathrm{m} .{ }^{2}\right)}{\mathrm{BSA}}$} & \multirow[t]{2}{*}{ Procedure } & \multirow[t]{2}{*}{$\begin{array}{l}\text { Heart } \\
\text { rate/ } \\
\text { min. }\end{array}$} & \multicolumn{3}{|c|}{$\begin{array}{c}\text { Brachial arterial } \\
\text { pressure (mm. Hg) }\end{array}$} & \multirow[t]{2}{*}{$\begin{array}{c}\text { Cardiac } \\
\text { output } \\
(1 . / \mathrm{min} .)\end{array}$} & \multirow[t]{2}{*}{$\begin{array}{c}\text { Stroke } \\
\text { volume } \\
\text { (ml.) }\end{array}$} & \multirow{2}{*}{$\begin{array}{l}\text { Total } \\
\text { periph. } \\
\text { vasc. } \\
\text { resist. }\end{array}$} & \multicolumn{2}{|c|}{$\begin{array}{c}\text { Plasma } \\
\text { catecholamines } \\
(\mu \mathrm{g} . / 1 .)\end{array}$} & \multirow[t]{2}{*}{$\begin{array}{l}\text { Flight } \\
\text { errors }\end{array}$} \\
\hline & & & & & Syst. & Diast. & Mean & & & & Epineph. & $\begin{array}{c}\text { Nor- } \\
\text { epineph. }\end{array}$ & \\
\hline 10 & 26 & 1.80 & $\begin{array}{l}\text { Rest } \\
\text { Flight } \\
\text { Recovery } \\
\text { Propranolol } \\
\text { Rest } \\
\text { Flight } \\
\text { Recovery }\end{array}$ & $\begin{array}{r}82 \\
110 \\
88 \\
65 \\
70 \\
64\end{array}$ & $\begin{array}{l}124 \\
159 \\
142 \\
144 \\
151 \\
136\end{array}$ & $\begin{array}{r}79 \\
110 \\
97 \\
99 \\
106 \\
94\end{array}$ & $\begin{array}{r}98 \\
139 \\
122 \\
121 \\
125 \\
113\end{array}$ & $\begin{array}{l}6 \cdot 2 \\
8 \cdot 3 \\
5 \cdot 5 \\
3 \cdot 5 \\
3 \cdot 9 \\
3 \cdot 7\end{array}$ & $\begin{array}{l}82 \\
76 \\
66 \\
57 \\
55 \\
60\end{array}$ & $\begin{array}{l}1.5 \\
1.7 \\
2.2 \\
3.5 \\
3.2 \\
3.0\end{array}$ & $\leqq 0 \cdot 1$ & $\begin{array}{l}0.6 \\
0.4\end{array}$ & 3 \\
\hline 11 & 46 & 1.90 & $\begin{array}{l}\text { Rest } \\
\text { Flight } \\
\text { Recovery } \\
\text { Exercise: I* } \\
\text { Recovery } \\
\text { Propranolol } \\
\text { Rest } \\
\text { Flight } \\
\text { Recovery } \\
\text { Exercise: I } \\
\text { Exercise: IIt } \\
\text { Recovery }\end{array}$ & $\begin{array}{r}78 \\
87 \\
83 \\
119 \\
87 \\
65 \\
69 \\
69 \\
62 \\
107 \\
125 \\
65\end{array}$ & $\begin{array}{l}122 \\
132 \\
118 \\
147 \\
123 \\
121 \\
133 \\
116 \\
129 \\
146 \\
122\end{array}$ & $\begin{array}{l}70 \\
77 \\
71 \\
75 \\
75 \\
74 \\
81 \\
69 \\
68 \\
71 \\
67\end{array}$ & $\begin{array}{r}93 \\
100 \\
93 \\
102 \\
98 \\
94 \\
94 \\
102 \\
90 \\
95 \\
102 \\
88\end{array}$ & $\begin{array}{r}5 \cdot 2 \\
5 \cdot 3 \\
4 \cdot 1 \\
7 \cdot 0 \\
\\
3 \cdot 1 \\
3 \cdot 6 \\
2 \cdot 8 \\
7 \cdot 5 \\
10 \cdot 5\end{array}$ & $\begin{array}{l}70 \\
62 \\
52 \\
55 \\
\\
48 \\
51 \\
52 \\
66 \\
82\end{array}$ & $\begin{array}{l}1.8 \\
1.9 \\
2.3 \\
1.5 \\
\\
3.0 \\
2.8 \\
3.0 \\
1.3 \\
1.0\end{array}$ & $\leqq 0 \cdot 1$ & $\begin{array}{l}0.5 \\
0.8\end{array}$ & 3 \\
\hline 12 & 41 & 1.80 & $\begin{array}{l}\text { Rest } \\
\text { Flight } \\
\text { Recovery } \\
\text { Exercise: I } \\
\text { Recovery } \\
\text { Propranolol } \\
\text { Rest } \\
\text { Flight } \\
\text { Recovery } \\
\text { Exercise: I } \\
\text { Exercise: II } \\
\text { Recovery }\end{array}$ & $\begin{array}{r}85 \\
94 \\
81 \\
116 \\
86 \\
\\
68 \\
73 \\
68 \\
102 \\
121 \\
78\end{array}$ & $\begin{array}{l}137 \\
157 \\
137 \\
162 \\
131 \\
125 \\
156 \\
129 \\
141 \\
156 \\
123 \\
\end{array}$ & $\begin{array}{r}79 \\
97 \\
83 \\
89 \\
85 \\
81 \\
102 \\
82 \\
77 \\
79 \\
77\end{array}$ & $\begin{array}{r}102 \\
118 \\
106 \\
122 \\
109 \\
99 \\
124 \\
101 \\
108 \\
116\end{array}$ & $\begin{array}{r}6.3 \\
6.6 \\
4.9 \\
10.0 \\
\\
3.9 \\
4.9 \\
3.9 \\
9.6 \\
15.3\end{array}$ & $\begin{array}{r}79 \\
73 \\
64 \\
84 \\
\\
\\
59 \\
64 \\
59 \\
93 \\
122\end{array}$ & $\begin{array}{l}1.6 \\
1.8 \\
2.1 \\
1.2 \\
\\
2.5 \\
2.6 \\
2.5 \\
1.2 \\
0.8\end{array}$ & $\begin{array}{r}0.2 \\
\leqq 0.1 \\
\leqq 0.1 \\
\leqq 0.1\end{array}$ & $\begin{array}{l}0.2 \\
0.7 \\
0.3 \\
0.4\end{array}$ & 3 \\
\hline 13 & 30 & $2 \cdot 19$ & $\begin{array}{l}\text { Rest } \\
\text { Flight } \\
\text { Recovery } \\
\text { Exercise: I } \\
\text { Recovery } \\
\text { Propranolol } \\
\text { Rest } \\
\text { Flight } \\
\text { Recovery } \\
\text { Exercise: I } \\
\text { Exercise: II } \\
\text { Recovery }\end{array}$ & $\begin{array}{r}69 \\
103 \\
79 \\
127 \\
84 \\
74 \\
71 \\
81 \\
68 \\
108 \\
130\end{array}$ & $\begin{array}{l}146 \\
161 \\
136 \\
150 \\
127 \\
121 \\
152 \\
122 \\
143 \\
152\end{array}$ & $\begin{array}{r}90 \\
99 \\
92 \\
96 \\
85 \\
\\
88 \\
103 \\
85 \\
88 \\
90\end{array}$ & $\begin{array}{r}114 \\
126 \\
107 \\
115 \\
104 \\
101 \\
121 \\
98 \\
106 \\
110\end{array}$ & $\begin{array}{r}5 \cdot 5 \\
7.9 \\
4 \cdot 4 \\
8 \cdot 6 \\
\\
3 \cdot 8 \\
5.2 \\
3.7 \\
8 \cdot 8 \\
11 \cdot 6\end{array}$ & $\begin{array}{l}87 \\
76 \\
63 \\
69 \\
\\
54 \\
67 \\
55 \\
81 \\
89\end{array}$ & $\begin{array}{l}2.1 \\
1.6 \\
2.4 \\
1.3 \\
\\
2.6 \\
2.3 \\
2.6 \\
1.2 \\
0.9\end{array}$ & $\begin{array}{l}\leqq 0.1 \\
\leqq 0.1 \\
\leqq 0 \cdot 1 \\
\leqq 0.1\end{array}$ & $\begin{array}{l}0.2 \\
0.7 \\
0.2 \\
1.0\end{array}$ & 1 \\
\hline 14 & 49 & 1.96 & $\begin{array}{l}\text { Rest } \\
\text { Flight } \\
\text { Recovery } \\
\text { Exercise: I } \\
\text { Recovery } \\
\text { Propranolol } \\
\text { Rest } \\
\text { Flight } \\
\text { Recovery } \\
\text { Exercise: I } \\
\text { Exercise: II } \\
\text { Recovery }\end{array}$ & $\begin{array}{r}74 \\
92 \\
74 \\
105 \\
66 \\
59 \\
59 \\
67 \\
61 \\
98 \\
112\end{array}$ & $\begin{array}{l}131 \\
149 \\
130 \\
150 \\
125 \\
122 \\
145 \\
124 \\
146 \\
155\end{array}$ & $\begin{array}{l}79 \\
95 \\
82 \\
79 \\
74 \\
73 \\
94 \\
77 \\
79 \\
84\end{array}$ & $\begin{array}{r}99 \\
114 \\
99 \\
108 \\
95 \\
92 \\
110 \\
95 \\
104 \\
112\end{array}$ & $\begin{array}{l}4.8 \\
7.0 \\
4.7 \\
8.8 \\
\\
3.9 \\
4 \cdot 1 \\
3.5 \\
7.6 \\
8.6\end{array}$ & $\begin{array}{l}65 \\
76 \\
64 \\
84 \\
\\
66 \\
61 \\
57 \\
78 \\
77\end{array}$ & $\begin{array}{l}2 \cdot 1 \\
1 \cdot 6 \\
2 \cdot 1 \\
1 \cdot 2 \\
\\
2 \cdot 4 \\
2 \cdot 7 \\
2 \cdot 7 \\
1 \cdot 4 \\
1 \cdot 3\end{array}$ & $\begin{array}{l}\leqq 0 \cdot 1 \\
\leqq 0.1 \\
\leqq 0 \cdot 1 \\
\leqq 0.1\end{array}$ & $\begin{array}{l}0 \cdot 3 \\
0 \cdot 4 \\
0 \cdot 4 \\
0 \cdot 4\end{array}$ & 3 \\
\hline 15 & 43 & 1.95 & $\begin{array}{l}\text { Rest } \\
\text { Flight } \\
\text { Recovery } \\
\text { Exercise } \\
\text { Recovery } \\
\text { Propranolol } \\
\text { Rest } \\
\text { Flight } \\
\text { Recovery } \\
\text { Exercise: I } \\
\text { Exercise: II } \\
\text { Recovery }\end{array}$ & $\begin{array}{r}65 \\
86 \\
63 \\
106 \\
63 \\
56 \\
65 \\
55 \\
93 \\
112 \\
60\end{array}$ & $\begin{array}{l}174 \\
191 \\
174 \\
201 \\
172 \\
165 \\
182 \\
162 \\
188 \\
200\end{array}$ & $\begin{array}{r}96 \\
104 \\
97 \\
106 \\
94 \\
97 \\
106 \\
95 \\
98 \\
104\end{array}$ & $\begin{array}{l}125 \\
140 \\
124 \\
139 \\
124 \\
125 \\
136 \\
121 \\
133 \\
141\end{array}$ & $\begin{array}{r}4.5 \\
6.3 \\
3.5 \\
8.5 \\
\\
2.8 \\
3.7 \\
3.1 \\
8.5 \\
10.2\end{array}$ & $\begin{array}{l}69 \\
74 \\
55 \\
81 \\
\\
50 \\
57 \\
56 \\
91 \\
91\end{array}$ & $\begin{array}{l}2.8 \\
2.2 \\
3.5 \\
1.6 \\
\\
4.5 \\
3.7 \\
3.9 \\
1.6 \\
1.4\end{array}$ & $\begin{array}{l}0.2 \\
0.7\end{array}$ & $\begin{array}{l}0 \cdot 3 \\
0 \cdot 4\end{array}$ & 1 \\
\hline
\end{tabular}

^ Exercise: I (load $600 \mathrm{kpm} / \mathrm{min}$.).

t Exercise: II (load $900 \mathrm{kpm} / \mathrm{min}$.). 


\section{RESULTS}

In each individual subject, numerous recordings of intra-arterial pressure and heart rate were made during each period. For purposes of comparison and calculation, the mean of all readings taken in any one period was calculated for each parameter, and reported in Table I. Similarly, as duplicate estimations of cardiac output were made, the average is reported.

I: Circulatory Response to Flight (15 pilots) (Table I). The heart rate varied at rest between 55 and 86 beats a minute, mean 73 . In flight the heart rate rose to between 71 and 120 beats a minute, mean $92(p<0.001)$. During recovery the rate decreased to a mean of 76 (range 59 to 96 ) beats per minute.

The mean brachial arterial pressure varied at rest between 78 and $137 \mathrm{~mm}$. $\mathrm{Hg}$, average 106. On flight the pressure rose to between 100 and $162 \mathrm{~mm}$. $\mathrm{Hg}$, mean $126(\mathrm{p}<0.001)$. On recovery the mean pressure fell (range and mean in 13 pilots: 93-144, and $115 \mathrm{~mm} . \mathrm{Hg}$, respectively).

The systolic and diastolic arterial pressures behaved similarly to the mean pressure. The pulse pressure increased approximately 10 per cent during flight.

The cardiac output varied at rest between 3.9 and $8.01 . / \mathrm{min}$., mean $5 \cdot 5$. On flight it rose to between 5.2 and $14 \mathrm{l} / \mathrm{min}$., mean $8.1 \quad(\mathrm{p}<0.001)$. During recovery it fell to between 3.5 and 10.2 , averaging $5.51 . / \mathrm{min}$.

The stroke volume ranged at rest between 59 and $112 \mathrm{ml}$. per beat, mean 78 . On flight it rose in eight subjects, fell in four subjects, and remained essentially unaltered in the remaining three pilots. The mean stroke volume during flight was $87 \mathrm{ml}$. The rise was not significant. On recovery the stroke volume decreased in all but one of the subjects.

The calculated peripheral vascular resistance varied at rest between $1 \cdot 1$ and 3.4 units

$$
\left(1 \text { unit }=\frac{\text { Mean arterial pressure }(\mathrm{mm} . \mathrm{Hg})}{\text { Cardiac output }(1 . / \mathrm{min} .) 10}\right),
$$

mean 2.0. On flight it varied between 0.9 and 2.3 PR-units, average 1.7 , decreasing in most subjects $(\mathrm{p}-0.01)$. During recovery it rose to a mean of $2 \cdot 2$ units.

II: Circulatory Response to Second Flight (following saline infusion) (4 pilots) (Table I and Fig. 3). The heart rate before the second flight period averaged 65 beats a minute. On flight it rose to an average of 79 . The increase ( 14 beats $/ \mathrm{min}$.) was similar to that ( 18 beats $/ \mathrm{min}$.) of the first period.
The mean brachial arterial pressure before the second flight period averaged $112 \mathrm{~mm}$. $\mathrm{Hg}$. On flight and on recovery it changed in a way similar to that in the first flight.

The systolic and diastolic pressures responded similarly to the two flights. The pulse pressure, also, showed approximately the same increase during the two flight periods.

The cardiac output during the second flight period was measured in three pilots. It rose to the same level as that obtained in the initial flight period.

The stroke volume rose to the same level on both flight occasions.

The calculated peripheral vascular resistance behaved in a similar manner on both flight occasions, showing a fall during flight and a rise on recovery.

III: Comparison of Circulatory Responses to Flight and to Subsequent Exercise (5 pilots) (Table I, Fig. 5). The heart rate, varying between 86 and 103 beats a minute on flight, rose further on exercise to between 105 and 127. The means were 92 and 114 beats a minute, respectively.

The mean brachial arterial pressure, varying between 100 and 140 (mean 120) mm. $\mathrm{Hg}$ on flight, also increased on exercise to between 102 and 139 (mean 117) $\mathrm{mm}$. Hg.

The arterial pulse pressure, varying between 54 and $87 \mathrm{~mm}$. $\mathrm{Hg}$ on flight, rose somewhat further on exercise to between 54 and $95 \mathrm{~mm}$. Hg. The means were 64 and $73 \mathrm{~mm}$. $\mathrm{Hg}$, respectively.

The cardiac output varying between 5.3 and 7.9 1 ./min. on flight, rose further on exercise to between 7.0 and $10.01 . / \mathrm{min}$. The means were 6.6 and 8.6 1./min., respectively.

The stroke volume, varying between 62 and $76 \mathrm{ml}$. on flight, increased to a similar level during exercise (between 55 and $84 \mathrm{ml}$.). The means were 72 and $75 \mathrm{ml}$., respectively.

The calculated peripheral vascular resistance, varying between 1.6 and 2.2 PR-units on flight, decreased further on exercise to between 1.2 and 1.6 units. The means were 1.8 and 1.4 units respectively.

IV: Influence of Propranolol on Response to Flight (11 pilots) (Tables I and II, Fig. 4). The heart rate measured at rest decreased. The average fall was 12 beats a minute. On flight the rise was of significantly smaller magnitude, as was the mean level achieved (70 as compared to 96 beats a minute before propranolol).

The brachial arterial mean pressure measured at rest was essentially unchanged after propranolol and the response to flight was in essence similar to that before the administration of propranolol. 

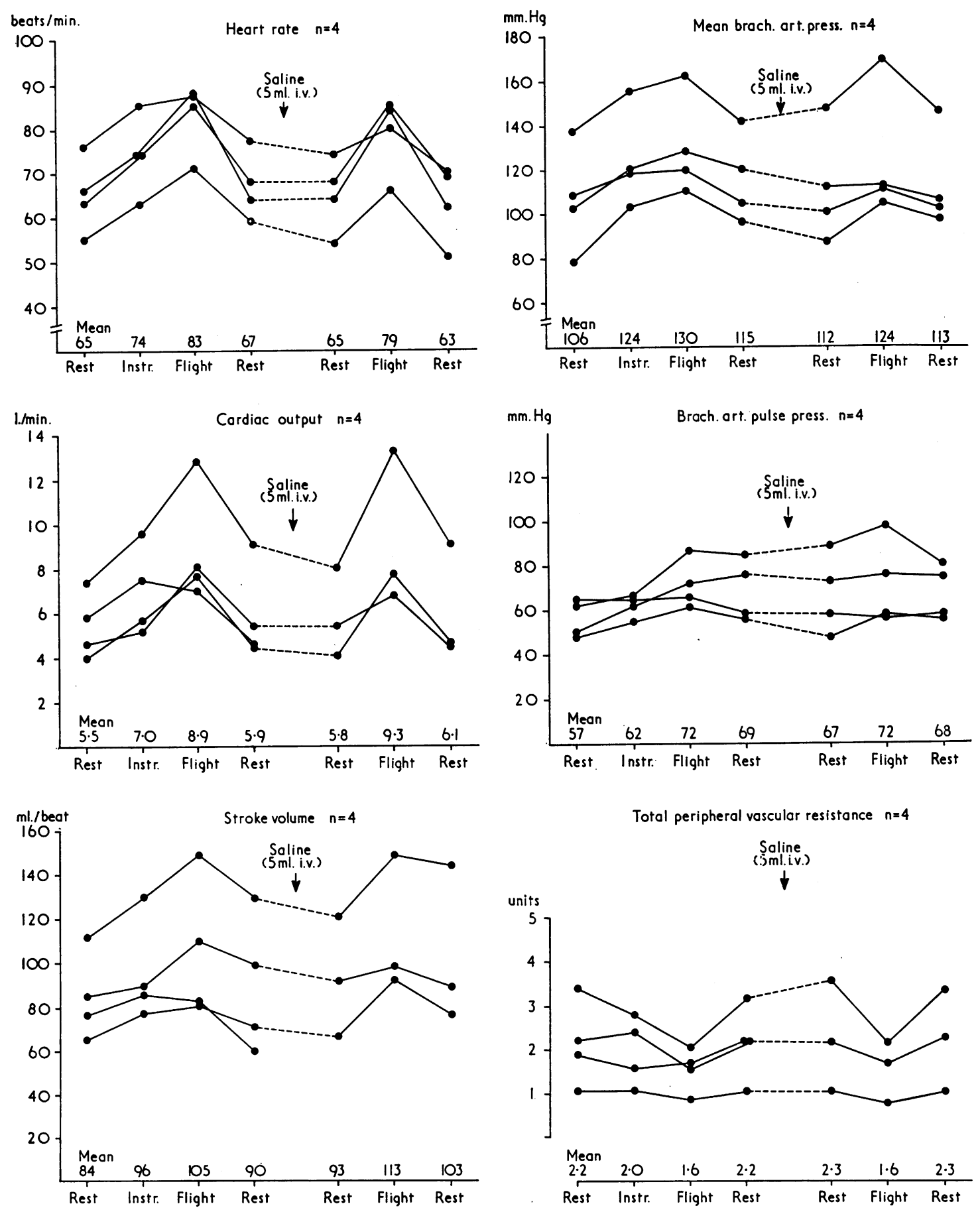

FIG. 3. - This graph shows the changes in heart rate, cardiac output, stroke volume, mean brachial arterial pressure, arterial pulse pressure, and calculated total peripheral vascular resistance at rest, following instructions and during flight in 4 pilots. The flight has been repeated after the intravenous administration of $5 \mathrm{ml}$. saline. 

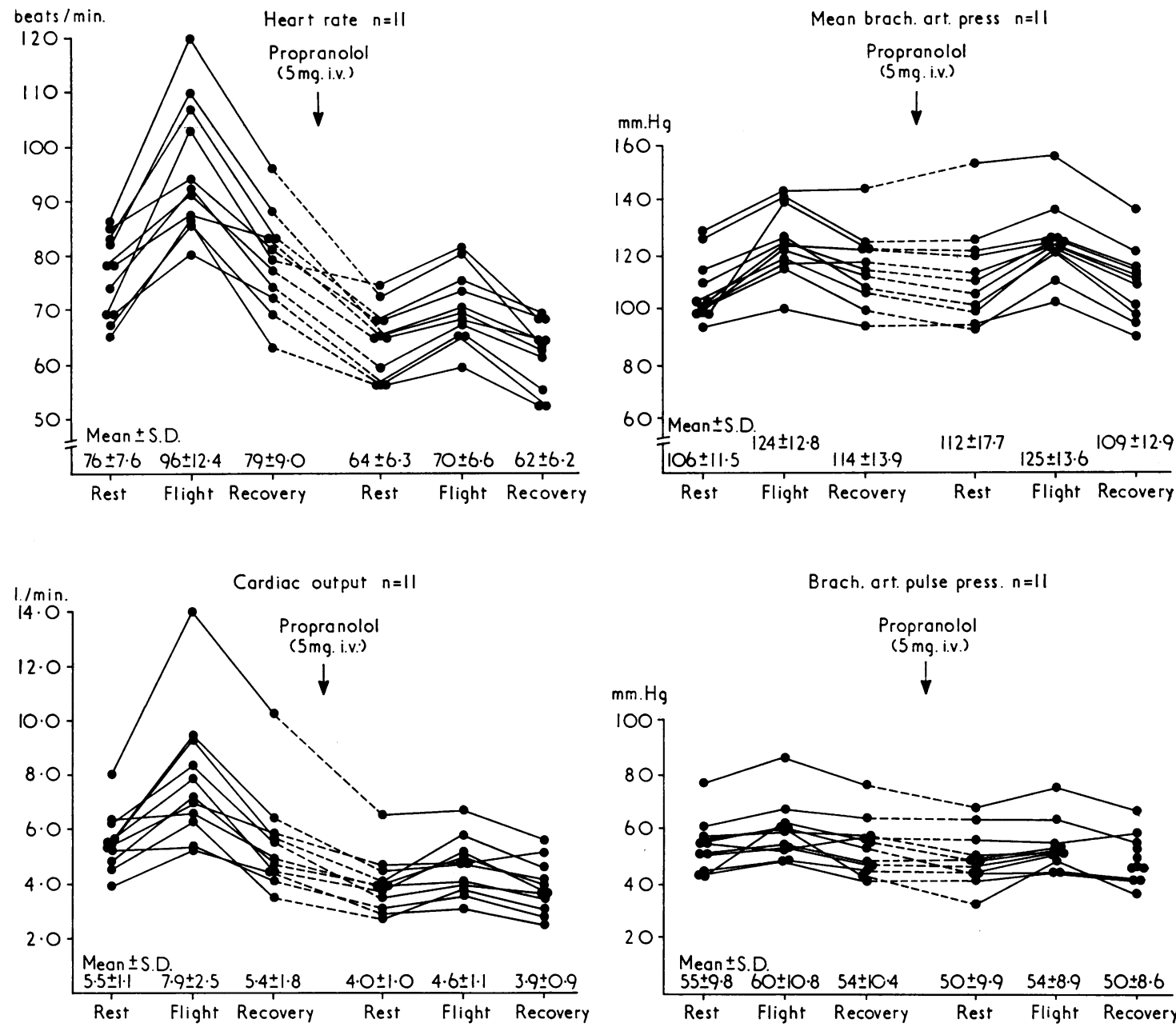

Brach. ort. pulse press. $n=11$

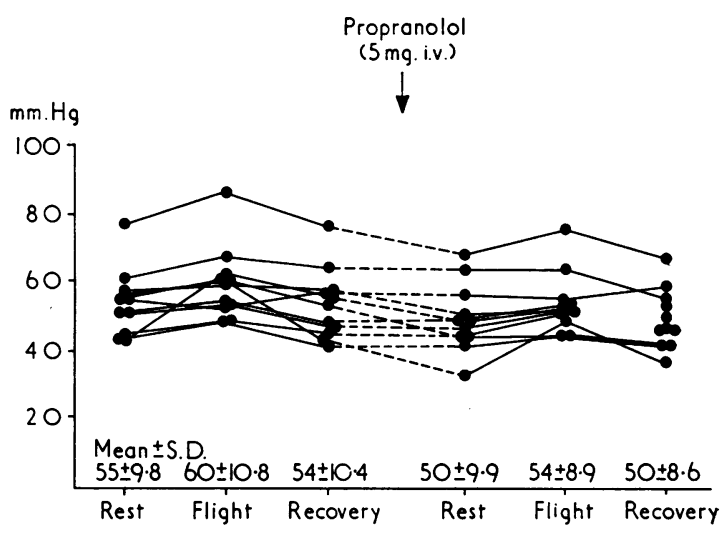

Stroke volume $n=11$
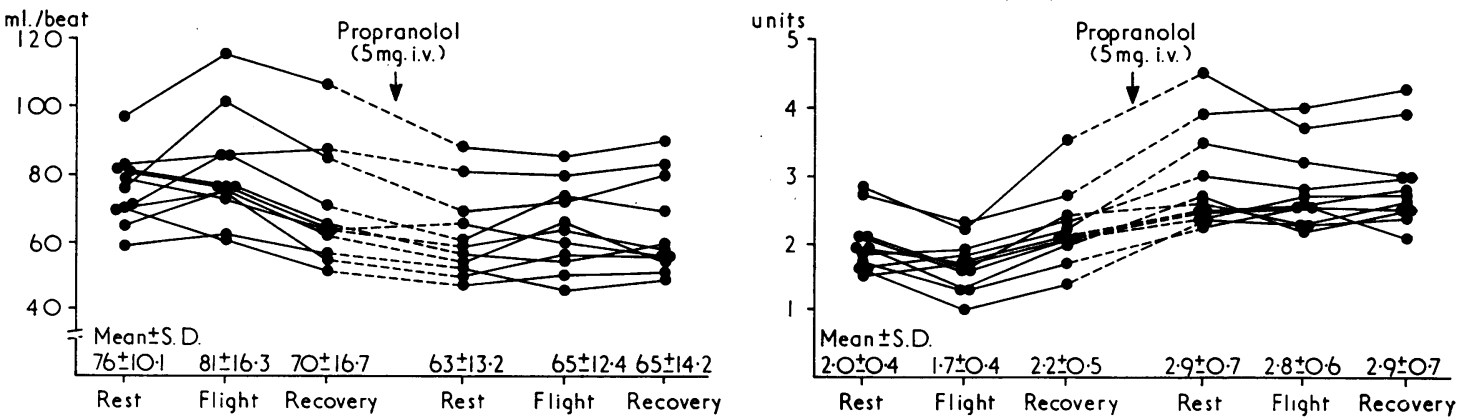

FIG. 4.-This graph shows the changes in heart rate, cardiac output, stroke volume, mean brachial arterial pressure, arterial pulse pressure, and calculated total peripheral vascular resistance at rest and during flight in 11 pilots (No. 5-15). The flight was repeated after the intravenous administration of $5 \mathrm{ml}$. propranolol. 
TABLE II

MEAN, STANDARD DEVIATION, AND SIGNIFICANCE OF DIFFERENCES CALCULATED IN 11 SUBJECTS

\begin{tabular}{|c|c|c|c|c|c|c|c|c|c|c|c|c|c|c|c|c|c|c|}
\hline \multirow{2}{*}{$\mathrm{n}_{\mathrm{d}}=11$} & \multicolumn{3}{|c|}{ Heart rate/min. } & \multicolumn{3}{|c|}{$\begin{array}{c}\text { Brachial artery } \\
\text { mean pressure } \\
(\mathrm{mm} . \mathrm{Hg})\end{array}$} & \multicolumn{3}{|c|}{$\begin{array}{l}\text { Pulse pressure } \\
(\mathrm{mm} . \mathbf{H g})\end{array}$} & \multicolumn{3}{|c|}{$\begin{array}{c}\text { Cardiac output } \\
(1 . / \mathrm{min} .)\end{array}$} & \multicolumn{3}{|c|}{$\begin{array}{l}\text { Stroke volume } \\
(\mathrm{ml} .)\end{array}$} & \multicolumn{3}{|c|}{$\begin{array}{l}\text { Total peripheral } \\
\text { resistance }\end{array}$} \\
\hline & $\overline{\mathbf{x}}_{\mathbf{d}}$ & S.D. & $\mathbf{p}$ & $\bar{\lambda}_{d}$ & S.D. & $\mathbf{p}$ & $\overline{\mathbf{x}}_{\mathbf{d}}$ & S.D. & $\mathbf{p}$ & $\overline{\mathbf{x}}_{\mathrm{d}}$ & S.D. & $\mathbf{p}$ & $\overline{\mathbf{x}}_{\mathbf{d}}$ & S.D. & p & $\overline{\mathbf{x}}_{\mathbf{d}}$ & S.D. & $\mathbf{p}$ \\
\hline $\begin{array}{l}\mathbf{F}_{1}-\mathbf{R}_{1} \\
\mathbf{F}_{2}-\mathbf{R}_{2} \\
\mathbf{R}_{2}-\mathbf{R}_{1} \\
\mathbf{F}_{2}-\mathbf{F}_{1}\end{array}$ & $\begin{array}{r}19.8 \\
6.2 \\
-12.0 \\
-25.7\end{array}$ & \begin{tabular}{|l|}
$9 \cdot 2$ \\
$2 \cdot 1$ \\
$6 \cdot 2$ \\
$8 \cdot 3$
\end{tabular} & $\begin{array}{l}0.001 \\
0.001 \\
0.001 \\
0.001\end{array}$ & $\begin{array}{r}17.6 \\
13.0 \\
5.5 \\
0.7\end{array}$ & $\begin{array}{r}8.4 \\
7.7 \\
12.5 \\
7.3\end{array}$ & $\begin{array}{l}0.001 \\
0.001 \\
0.20 \\
0.80\end{array}$ & $\begin{array}{r}5.2 \\
4.1 \\
-4.9 \\
-6.0\end{array}$ & \begin{tabular}{|l|}
$5 \cdot 5$ \\
$5 \cdot 4$ \\
$8 \cdot 1$ \\
$4 \cdot 5$ \\
\end{tabular} & $\begin{array}{l}0.02 \\
0.05 \\
0.10 \\
0.005\end{array}$ & $\begin{array}{r}2.4 \\
0.6 \\
-1.6 \\
-3.3\end{array}$ & $\begin{array}{l}1.7 \\
0.5 \\
0.7 \\
1.7\end{array}$ & $\begin{array}{l}0.005 \\
0.005 \\
0.001 \\
0.001\end{array}$ & $\begin{array}{r}5.5 \\
2.6 \\
-9.7 \\
-15.4\end{array}$ & $\begin{array}{r}10.9 \\
6-7 \\
13.7 \\
8.3\end{array}$ & & $\begin{array}{r}-0.3 \\
-0.1 \\
1.0 \\
1.1\end{array}$ & $\begin{array}{l}0.3 \\
0.3 \\
0.5 \\
0.4\end{array}$ & $\begin{array}{l}0.02 \\
0.30 \\
0.001 \\
0.001\end{array}$ \\
\hline
\end{tabular}

$\overline{\mathbf{X}} \mathrm{d}=$ Mean; S.D. $=$ standard deviation.

$R_{1}=$ rest, and $F_{1}=$ flight, before propranolol; $R_{2}=$ rest, and $F_{2}=$ flight after propranolol.

The brachial arterial pulse pressure following propranolol was unaltered at rest but the response in flight was significantly lower than that recorded during the first flight period.

The cardiac output at rest decreased significantly after propranolol. The average decrease was 1.6 litres per minute. On flight it rose, but to a much smaller extent than during the first flight period. The mean rise after propranolol was 0.6 as compared to $2.41 . / \mathrm{min}$. during the initial flight.

The stroke volume decreased somewhat at rest following propranolol, and was essentially unchanged in the subsequent flight period from this new level.

The calculated peripheral resistance at rest rose markedly after propranolol in all pilots (mean 3.0 PR-units). A minor decrease of the resistance was observed during the second flight period (mean 2.8 PR-units).

$V:$ Comparison of Influence of Propranolol on Response to Flight and Exercise (5 pilots) (Table I, Fig. 5). The heart rate response to flight following propranolol was smaller than that observed before the administration of the drug. The increase observed during exercise reached a lower level after propranolol. The mean rise on flight was only 7 beats a minute as compared to 18 beats a minute before propranolol. The corresponding figures on exercise were 39 and 38 beats a minute, respectively. On the higher exercise load applied after propranolol a further rise in heart rate occurred.

The mean brachial arterial pressure was little influenced by propranolol. The rises occurring both on flight and on exercise were of similar magnitude to those before the injection. A further slight rise was recorded during the higher exercise load applied after propranolol.

The brachial arterial pulse pressure at rest decreased to a slightly lower level after propranolol, and was likewise little influenced in its response to flight as well as to exercise.
The cardiac output had decreased conspicuously at rest after propranolol and the rise on flight was less than that observed before the injection of the drug. On subsequent exercise there was a significant rise in cardiac output to a level similar to that seen before propranolol. A further increase occurred on the higher exercise load after propranolol.

The stroke volume was lower at rest after propranolol. On flight it rose but did not reach the same level as that achieved before the propranolol injection. On exercise there was a consistent and much larger rise as compared to that observed before propranolol.

The calculated total peripheral vascular resistance, much increased after propranolol, showed little change on the subsequent flight and then fell during exercise to the same level as that seen with exercise before propranolol. On the higher exercise load a further decrease was recorded.

VI: Arterial Plasma Levels of Epinephrine and Norepinephrine (Table I). Arterial samples for catecholamine analyses were taken up to four times in twelve subjects. A mean norepinephrine plasma concentration of $0.6 \mu \mathrm{g} . / 1$. was measured at rest. The mean level increased to $0.8 \mu \mathrm{g}$. $/ 1$. during flight, an increase being found in six of the ten pilots. Detectable amounts of plasma epinephrine were found in only two pilots. On physical exercise $(600 \mathrm{kpm} / \mathrm{min}$.) the norepinephrine level increased similarly to that during flight. In the five pilots investigated, the mean concentration amounted to $0.3 \mu \mathrm{g}$. $/ 1$. at rest and $0.6 \mu \mathrm{g}$. $/ 1$. on exercise. Plasma epinephrine was detected in only one pilot.

VII: Record of Flying Ability and Number of Errors Committed During Flight (Table I). This record was kept in 14 studies. It was found that on an average the pilots performed equally well on the two flight occasions; improvement on the second flight occasion was registered in only four subjects. 

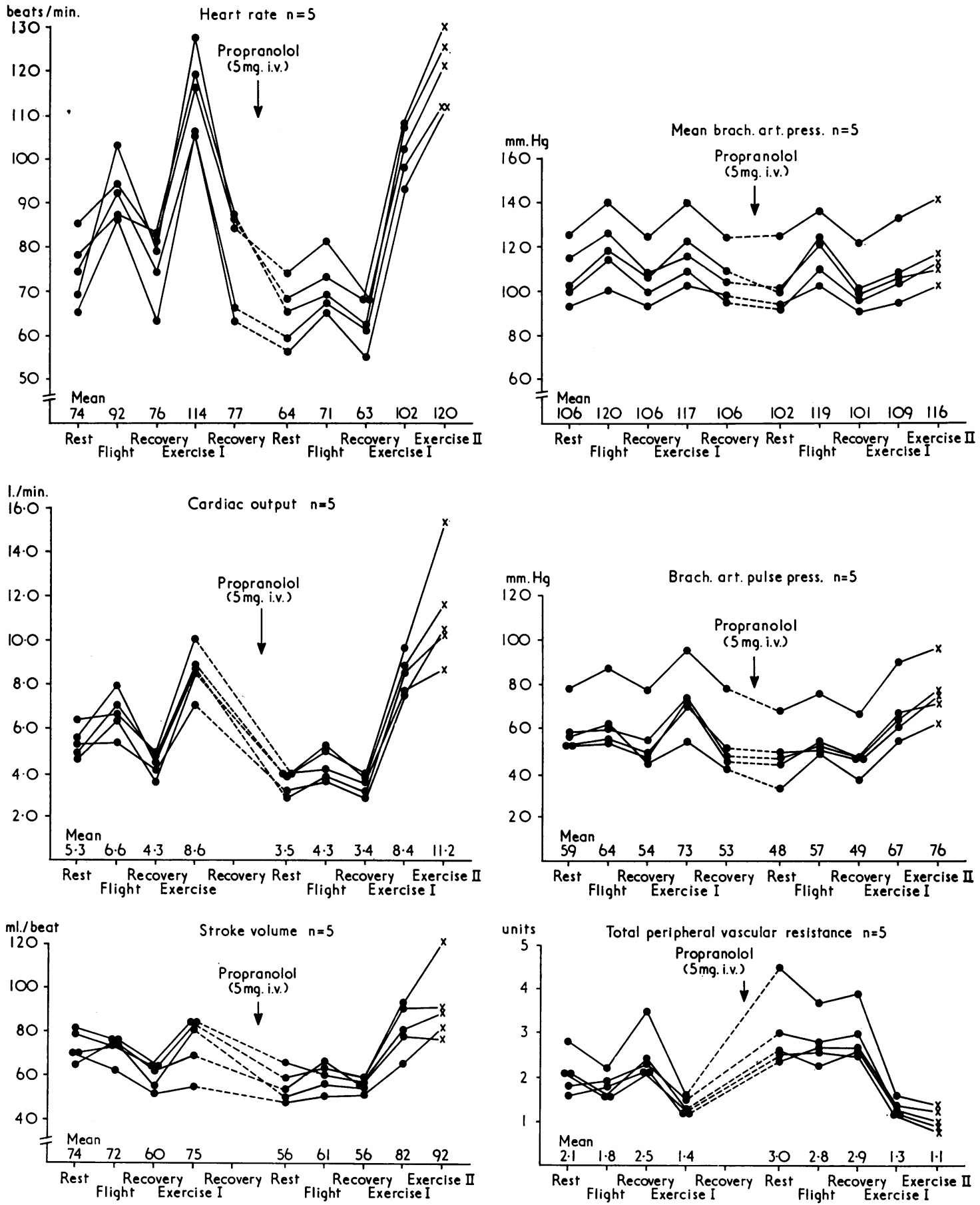

FIG. 5.-This graph shows the changes in heart rate, cardiac output, stroke volume, mean brachial arterial pressure, arterial pulse pressure, and calculated total peripheral vascular resistance at rest, during flight, and on subsequent bicycle ergometry in 5 pilots (No. 11-15). The flight and the bicycle ergometry were repeated after the intravenous administration of $5 \mathrm{ml}$. propranolol. On "Exercise I" the ergometer load was 600 kpm/min.; on "Exercise II" the load was $900 \mathrm{kpm} / \mathrm{min}$. 


\section{Discussion}

One of the major aims of the present investigation was to create a suitable experimental model for the study of the hæmodynamic response to an induced stressful situation in man. Link trainer simulated flight, applied to pilots, was found to be an appropriate stressor and to produce a consistent hæmodynamic response pattern. The reaction was quantitatively reproduceable as judged from the response observed on a second flight period, being similar to that during the initial flight. In analysing the errors committed by the pilot during flight it was found that these, and probably therefore the degree of difficulty with which the pilot had to cope, were of a similar order on both occasions. The use of a closed cockpit allowed him to be in contact with the outside by telephone only. One of the major advantages of this arrangement was that the hæmodynamic measurements could be made at times unknown to the subject. The study was performed with the pilot in the sitting position, allowing for the ordinarily cccurring regional shifts of blood flow. The degree of muscle activity involved in operating the Link trainer during flight was small and probably of no major importance in the present investigation.

The pilots, when exposed to the demanding situation of flying the Link trainer, displayed a rise in the mean systemic blood pressure, associated with an increased heart rate and cardiac output, respectively. The calculated peripheral vascular resistance was generally slightly lower. The response pattern is in accordance with that suggested or shown by others using different emotional stimuli (Grollman, 1929; Stead et al., 1945; Hickam et al., 1948; Stevenson et al., 1949; Wolf et al., 1955; Brod, 1963).

The circulatory responses to physical exercise were the same as those earlier observed in the sitting posture (Bevegård, Holmgren, and Jonsson, 1960), i.e. a conspicuous rise in the heart rate and the cardiac output and a moderate increase in the systemic blood pressure. Although the exercise load $(600 \mathrm{kpm} / \mathrm{min}$.) was relatively moderate, corresponding to an oxygen consumption of 1.5 litres per minute (Åstrand, 1960), the response of both the heart rate and the cardiac output were more pronounced than during the stress of flight, while the blood pressure increment was somewhat less.

The difference in the hæmodynamic reaction between that of emotional stress and that of physical exercise is mainly due to the metabolically induced skeletal muscle vasodilatation occurring in the latter situation. Sympathetic stimuli, causing augmented heart performance and vasoconstriction in both the splanchnic and the cutaneous vascular bed, occur in both situations (Brod, 1963). These cardiac and vascular responses are mediated by different types of adrenergic receptors, beta and alpha respectively (Ahlquist, 1948), and can thus be available for separate pharmacological analyses. The consistency in the hæmodynamic responses to both flight and exercise also provided an important requisite for evaluating the effects of an administered pharmacological agent in these situations. In an attempt to analyse the contribution of beta adrenergic stimulation, beta receptor blockade was produced by intravenous propranolol. The conspicuously reduced or eliminated circulatory response to isoprenaline after propranolol confirmed the efficiency of the beta receptor blocking action.

Already at rest, propranolol had induced significant cardiovascular effects. The resting heart rate decreased, presumably as a result of blockade of the positive chronotropic activity present already before flight. The cardiac output decreased while the systemic arterial pressure remained unchanged. The concomitant increase in calculated total peripheral resistance suggests that relative vasoconstriction had occurred. Three hypotheses may be advanced to explain this finding. First, previously present beta-adrenergic vasodilatation, presumably epinephrine mediated, may have been abolished (Glover, Greenfield, and Shanks, 1962). The occasional finding of detectable amounts of epinephrine in plasma, normally not present, supports this suggestion. However, the inconsistency with which epinephrine was demonstrated, despite the uniformity of the peripheral resistance response, indicates that this can be only a partial explanation. Secondly, it might be postulated that the increase in resistance reflects a direct effect of propranolol on the vessels. No such sympathomimetic properties of propranolol have been demonstrated in animal studies (Black et al., 1965). Thirdly, a reflex mechanism must be considered. For, in the face of such profound falls in cardiac output, if a degree of vasoconstriction were not to occur, severe hypotension would result. Any tendency towards hypotension would stimulate vasoconstrictor nerve activity mediated via the baroreceptors.

During flight after propranolol, the increase in both heart rate and cardiac output was much smaller than that observed in the pre-propranolol study, which indicates that the beta adrenergic system is very involved in the hæmodynamic response to emotional stress. Adaptation to the repeated flight procedure did, as shown, apparently not play a major role.

During physical exercise the cardiac output and the blood pressure rose to approximately the same levels as before the receptor blockade. The heart 
rate response was less after propranolol than before its administration, and consequently the stroke volume increased. The finding of a further rise in the cardiac output recorded on the subsequent higher exercise load indicates that beta adrenergic stimulation of the heart did not play a major role in mediating the cardiovascular response. Similar findings have been reported by others using nethalide (Chamberlain and Howard, 1964; Segel and Bishop, 1965; Schröder and Werkö, 1965) or propranolol (Epstein et al., 1965).

It is striking that along with various types of emotional stress, even in connexion with flight (Euler and Lundberg, 1954), an increase in the urinary epinephrine excretion is an almost consistent finding. In the present investigation no increase in plasma epinephrine was observed. The arterial plasma catecholamine data can probably not be directly correlated to earlier observations on urinary catecholamine excretion. Detectable amounts of plasma epinephrine, normally not found (Carlsten et al., 1965), were present in only a few pilots. In contrast, the plasma norepinephrine levels were higher. The resting values were within or slightly higher than those reported earlier (Vendsalu, 1960; Chidsey, Harrison, and Braunwald, 1962; Carlsten et al., 1965). A slight rise was noted on flight as well as on exercise. Others have reported a similar increase of norepinephrine on exercise (Chidsey et al., 1962; Carlsten et al., 1965).

\section{SUMMARY}

Heart rate, systemic arterial blood pressure, and cardiac output, when measured in 15 pilots throughout a Link trainer simulated flight, all increased. This resulted in only a slight decrease in the peripheral vascular resistance.

A second Link trainer simulated flight after saline administration was performed by four of the pilots. Similar hæmodynamic changes were recorded as initially.

Eleven of the pilots went through a second Link trainer simulated flight after the intravenous administration of $5 \mathrm{mg}$. propranolol, a beta adrenergic blocking agent. While the responses in heart rate and diastolic blood pressure to injected isoprenaline were marked before the receptor blockade, only slight responses were observed after the blockade. During the preflight period after propranolol had been given, the heart rate and the cardiac output were significantly reduced while the arterial pressure was unchanged. Consequently, the peripheral vascular resistance had increased. During flight the increase in heart rate and cardiac output was significantly less as compared with the initial flight period. The augmented response in arterial blood pressure was not influenced by the beta adrenergic receptor blockade, and thus the peripheral vascular resistance remained increased.

In five of the eleven pilots to whom propranolol was given, physical exercise with the pilots sitting on a bicycle ergometer was also performed before and after the beta-receptor blockade. Increases in cardiac output and arterial blood pressure associated with conspicuous falls in peripheral vascular resistance occurred in both periods and to similar levels of each parameter. Effects of beta adrenergic blockade in this situation were seen as a lower level of heart rate was achieved.

The mean arterial plasma concentration of norepinephrine, measured in the majority of pilots, increased slightly during flight and during exercise. In contrast, the arterial plasma concentration of epinephrine was not measurable except in a few pilots.

The results indicate that beta adrenergic receptor activity is extensively involved in the circulatory reaction to emotional stress. In contrast, this activity appears to be involved but less essential in the achievement of the circulatory adjustments during moderate physical exercise.

The present investigation was made possible through the courtesy of Colonel Uno Lundberg, Surgeon General of the Royal Swedish Airforce. It was supported by the Swedish National Association against Heart and Lung Diseases and the Medical Defence Research Council. The assistance of Miss Birgit Ryd, Mr. C.-E. Lager, Mr. C. Norrbäck, Mr R. Forsberg, and Miss Viveca Sandberg is gratefully acknowledged. The Department of Clinical Physiology at the Serafimer Hospital also rendered valuable technical service.

\section{REFERENCES}

Ahlquist, R. P. (1948). A study of the adrenotropic receptors. Amer. F. Physiol., 153, 586.

Åstrand, I. (1960). Aerobic work capacity in men and women with special reference to age. Acta physiol. scand., 49, Suppl. 169.

Bevegård, S., Holmgren, A., and Jonsson, B. (1960). The effect of body position on the circulation at rest and during exercise, with special reference to the influence on the stroke volume. Acta physiol. scand., 49, 279.

Black, J. W., Crowther, A. F., Shanks, R. G., Smith, L. H., and Dornhorst, A. C. (1964). A new adrenergic betareceptor antagonist. Lancet, $1,1080$.

-, Duncan, W. A. M., and Shanks, R. G. (1965). Comparison of some properties of pronethalol and propranolol. Brit. F. Pharmacol., 25, 577.

Blair, D. A., Glover, W. E., Greenfield, A. D. M., and Roddie, I. C. (1959). Excitation of cholinergic vasodilator nerves to human skeletal muscles during emotional stress. F. Physiol. (Lond.), 148, 633.

Brod, J. (1963). Hæmodynamic basis of acute pressor reactions and hypertension. Brit. Heart F., 25, 227. 
—, Fencl, V., Hejl, Z., and Jirka, J. (1959). Circulatory changes underlying blood pressure elevation during acute emotional stress (mental arithmetic) in normotensive and hypertensive subjects. Clin. Sci., 18, 269.

Carlsten, A., Häggendal, J., Hallgren, B., Jagenburg, R., Svanborg, A., and Werkö, L. (1965). Effects of ganglionic blocking drugs on blood glucose, amino acids, free fatty acids and catecholamines at exercise in man. Acta physiol. scand., 64, 439.

Chamberlain, D. A., and Howard, J. (1964). The hæmodynamic effects of $\beta$-sympathetic blockade. Brit. Heart F., 26, 213.

Chidsey, C. A., Harrison, D. C., and Braunwald, E. (1962). Augmentation of the plasma nor-epinephrine response to exercise in patients with congestive heart failure. New Engl. f. Med., 267, 650.

Epstein, S. E., Robinson, B. F., Kahler, R. L., and Braunwald, E. (1965). Effects of beta-adrenergic blockade on the cardiac response to maximal and submaximal exercise in man. $\mathcal{f}$. clin. Invest., 44, 1745.

Euler, U. S. v., and Lundberg, U. (1954). Effect of flying on the epinephrine excretion in air force personnel. F. appl. Physiol., 6, 551.

Glover, W. E., Greenfield, A. D. M., and Shanks, R. G. (1962). The contribution made by adrenaline to the vasodilatation in the human forearm during emotional stress. f. Physiol. (Lond.), 164, 422.

Grollman, A. (1929). The effect of psychic disturbances on the cardiac output, pulse rate, blood pressure, and oxygen consumption of man. Amer. f. Physiol., 89, 584.

Häggendal, J. (1963). An improved method for fluorimetric determination of small amounts of adrenaline and nor- adrenaline in plasma and tissues. Acta physiol. scand., $59,242$.

Hickam, J. B., Cargill, W. H., and Golden, A. (1948). Cardiovascular reactions to emotional stimuli. Effect on the cardiac output, arteriovenous oxygen difference, arterial pressure, and peripheral resistance. $\mathcal{F}$. clin. Invest., 27, 290.

Holmgren, A. (1956). Circulatory changes during muscular work in man. With special reference to arterial and central venous pressures in the systemic circulation. Scand. F. clin. Lab. Invest., 8, Suppl. 24.

Schröder, G., and Werkö, L. (1965). Hemodynamic studies and clinical experience with nethalide, a beta-adrenergic blocking agent. Amer. F. Cardiol., 15, 58.

Segel, N., and Bishop, J. M. (1965). The circulatory effects of pronethalol with special reference to changes in heart rate and stroke volume during exercise. Clin. Sci., 29, 363.

Stead, E. A., Jr., Warren, J. V., Merrill, A. J., and Brannon, E. S. (1945). The cardiac output in male subjects as measured by the technique of right atrial catheterization. Normal values with observations on the effect of anxiety and tilting. f. clin. Invest., 24, 326.

Stevenson, I. P., Duncan, C. H., and Wolff, H. G. (1949). Circulatory dynamics before and after exercise in subjects with and without structural heart disease during anxiety and relaxation. f. clin. Invest., 28, 1535.

Vendsalu, A. (1960). Studies on adrenaline and noradrenaline in human plasma. Acta physiol. scand., 49, Suppl. 173.

Wolf, S., Cardin, P. V., Jr., Shepard, E. M., and Wolff, H. G. (1955). Life Stress and Essential Hypertension. Williams and Wilkins, Baltimore. 